\title{
Mineral trioxide aggregate a reliable alternative material for pulpotomy in primary molar teeth
}

\author{
Is mineral trioxide aggregate more effective than formocresol for pulpotomy \\ in primary molars?
}

\section{Aeinehchi M, Dadvand S, Fayazi S, Bayat-Movahed S.}

Randomized controlled trial of mineral trioxide aggregate and formocresol for pulpotomy in primary molar teeth. Int Endod J 2007; 40:261-267

Design This was a randomised controlled trial. Intervention The trial assessed application of formocresol (FC) or mineral trioxide aggregate (MTA) during pulpotomy in primary molar teeth.

Outcome measure Treatment was evaluated by recording clinical signs (spontaneous pain, swelling, pain on palpation or percussion and sinus tract formation) or radiological signs of failure (root resorption, periodontal ligament widening, and apical, lateral or furcal radiolucency).

Results No signs of clinical failure were observed at 3 and 6 months but, at the 6-month follow-up, significantly more cases (P 0.036) with root resorption were seen in the FC group. No cases of resorption occurred in the MTA group. The surrounding tissue showed radiographical signs of post-treatment disease in four FC-group members; none was seen with MTA use.

Conclusions After 6 months, pulpotomy with MTA was associated with fewer cases of root resorption and post-treatment disease. MTA appears to be a reliable alternative material for pulpotomy in primary molar teeth.
Address for correspondence: Mohamad Aeinehchi, Department of

Endodontics, Faculty of Dentistry, Azad University of Medical Sciences, Tehran, Iran. E-mail: ocrt@sina.tums.ac.ir

\section{Commentary}

This trial was designed to compare the use of MTA and FC for pulpotomies in primary molars. The study is in general very well reported. The conclusion was that there were fewer cases of root resorption or evidence of intraradicular infection in the MTA pulpotomy group (n, 0 out of 43) compared with the FC pulpotomy group (n, 11 out of 57). No tooth demonstrated clinical signs or symptoms of failure: all failures were detected radiographically.

Although the 6-month results presented are interesting, a longer trail period would be more valid. For example, to be included in the Cochrane Systematic Review on pulpotomy agents for primary teeth, at least 12 months follow-up is required. ${ }^{1}$ Clinicians need to know that success rates are in excess of 2-3 years. Hopefully the authors will report longer-term results.

Although this is a well-conducted study, there are some points that can be criticised. The FC arm was restored with either amalgam or glass ionomer and the MTA arm only with amalgam. It is likely that amalgam would be a more successful restorative material. In any case, it would have been preferable to place stainless steel crowns as these offer the best coronal seal and consequently the best chance of success. ${ }^{2}$

The randomisation was by child and, therefore, because some children had multiple pulpotomies, there was clustering. This was not allowed for in the analysis. A further criticism is that there is no intention to treat analysis, while 18 children were lost from the FC group and eight children from the MTA arm. These are not accounted for in any way. Questions also remain unanswered about the costeffectiveness of MTA since it is a relatively expensive material. How does it compare with ferric sulphate as a pulpotomy agent?

The study was conducted in early 2001 and this is the major problem of the study. Time has moved on. It is not clear why there has been such a delay in reporting this trial. The International Agency for Research on Cancer Working Group has since classed formaldehyde as a carcinogen. and most, if not all, Dental Schools in the United Kingdom have accordingly stopped teaching techniques using it. Having said this, the results of this study confirm other work indicating that use of MTA is a superior technique and clinicians who have not stopped using FC can change on the basis of evidence showing it to be more successful. ${ }^{2}$

\section{Chris Deery}

Department of Oral Health and Development, School of Clinical Dentistry, University of Sheffield, Sheffield, South Yorkshire, UK

1. Nadin G, Goel BR, Yeung CA, Glenny AM. Pulp treatment for extensive decay in primary teeth. Cochrane Database Syst Rev 2003; issue 1.

2. Eidelman E, Holan G, Fuks AB. Mineral trioxide aggregate vs formocresol in pulpotomised primary molars. Pediatric Dent 2001; 23:15-18.

Evidence-Based Dentistry (2007) 8, 107. doi:10.1038/sj.ebd.6400526 